\title{
Do COVID-19-Related Social Quarantine and Risk Perception Result in Persistent Behaviors Due to Changes in Human Brain Structures?
}

\author{
Ata Pourabbasi (iD) ${ }^{1,}$, Parastou Kordestani Moghadam ${ }^{2}$ and Sepideh Nikfarjam ${ }^{3}$ \\ ${ }^{1}$ Endocrinology and Metabolism Research Center, Endocrinology and Metabolism Clinical Sciences Institute, Tehran University of Medical Sciences, Tehran, IR Iran \\ ${ }^{2}$ Department of Cognitive Neuroscience, School of Nursing and Midwifery, Lorestan University of Medical Sciences, Khoramabad, IR Iran \\ ${ }^{3}$ Department of Medical Biotechnology, Faculty of Advanced Medical Sciences, Tabriz University of Medical Sciences, Tabriz, IR Iran \\ "Corresponding author: Endocrinology and Metabolism Research Center, Endocrinology and Metabolism Clinical Sciences Institute, Tehran University of Medical Sciences, \\ Tehran, IR Iran. Email: atapoura@gmail.com
}

Received 2020 May 13; Accepted 2020 June 13.

Keywords: Quarantine, Risk Perception, Risk-Taking, Gambling, Brain

\section{Dear Editor,}

The coronavirus disease 2019 (COVID-19) pandemic is one of the most significant pandemics that has affected all nations worldwide. Health care systems in all countries are currently struggling and dealing with this pandemic, and some of them have achieved remarkable successes in this respect (1). Among all incidents related to this global pandemic, two social factors, including quarantine and risk perception, require further consideration. Home quarantine for disease prevention and post-disease quarantine are the two preferred policies pursued by most countries to manage this situation (2). However, a fact that needs to be carefully inspected while adopting such policies is that long-term quarantine may lead to persistent alterations in neural structure. Several studies previously investigated the effects of long-term quarantine and social isolation on neural structure and function and reported several changes in brain structure such as the hippocampus and hypothalamus as well as modifications in the secretory pathways of some of neuromediators such as serotonin (3-5). These results suggest that implementing home quarantine policies to manage COVID-19 can lead to other complications in the future, considering the functional and structural changes in the brain. Moreover, the elevated prevalence of post-COVID-19 mental disorders may be partly attributed to these structural changes, validation of which requires further studies (6).

The outbreak of COVID-19 has resulted in excessive fear and panic in all societies. In other words, through the widespread activities of media around the world, societies have perceived the relative risk of the pandemic. Subsequently, numerous self-care behaviors are adopted as the result of a risk perception development during the COVID-19 outbreak. Conceivably, it has rarely happened that up to $90 \%$ of people in a community follow health protocols announced by health care systems, which indicates the perception of risk by societies (7). The association between neural structure, particularly prefrontal structure, and risk perception has been demonstrated in several other studies $(8,9)$. These studies concluded that a variety of risk perception-based behaviors, including selfcare behaviors, are established during pandemics as a result of the development of the corresponding neural structures, which is also one of the points that should be noticed during this particular pandemic. Therefore, if the risk perception triggered by a pandemic can cause the development of the corresponding neural structures, we can hope that through the cross-relation between risk perceptionbased behaviors, many other self-care behaviors such as eating habits or normal driving behavior are more likely to be institutionalized in communities during the postCOVID-19 period. Furthermore, the prevalence of high-risk behaviors, including high-risk sexual behaviors, addiction, and smoking, may be somehow reduced. All of these consequences can be attributed to the effects of COVID-19 pandemic on the neural structures corresponding to risk perception.

According to the mentioned points, it can be concluded that the two notable social phenomena during the COVID-19 pandemic, i.e. home quarantine or social isolation and the respective risk perception, can result in persistent influences on community behavior during the postCOVID-19 period through affecting neural structures. Of course, it should be noted that these impacts are separate from the direct effects of SARS-CoV-2 (Severe Acute Respira- 
tory Syndrome Coronavirus 2) on the brain, which can result in some neurological manifestations (10).

In conclusion, meticulous considerations of efficient future interventions to decrease home quarantine complications and help preserve self-care behaviors resulted from risk perception is an essential point that needs to be attended to by health care policymakers.

The authors are collecting other existing evidence to prove the association of quarantine and risk perception with the respective persistent alterations in neural structure, and the results will be published in the near future.

\section{Footnotes}

Authors' Contribution: Ata Pourabbasi designed and directed the project. All authors contributed to the implementation of the research and the final manuscript.

Conflict of Interests: The authors declare no conflict of interest.

Funding/Support: There is no funding support.

\section{References}

1. Raoofi A, Takian A, Sari AA, Olyaeemanesh A, Haghighi H, Aarabi M. COVID-19 pandemic and comparative health policy learning in Iran. Arch Iran Med. 2020;23(4):220-34. doi: 10.34172/aim.2020.02.
2. Lauer SA, Grantz KH, Bi Q, Jones FK, Zheng Q, Meredith HR, et al. The incubation period of coronavirus disease 2019 (COVID-19) from publicly reported confirmed cases: estimation and application. Ann Intern Med. 2020;10:M20-504. doi: 10.7326/M20-0504.

3. Cacioppo JT, Cacioppo S, Capitanio JP, Cole SW. The neuroendocrinology of social isolation. Annu Rev Psychol. 2015;66:733-67. doi: 10.1146/annurev-psych-010814-015240.

4. Krupina NA, Khlebnikova NN, Narkevich VB, Naplekova PL, Kudrin VS. The Levels of Monoamines and Their Metabolites in the Brain Structures of Rats Subjected to Two-and Three-Month-Long Social Isolation. Bull Exp Biol Med. 2020:1-5. doi: 10.1007/s10517-020-04761-5.

5. Tunbak H, Vazquez-Prada MC, Ryan TM, Kampff AR, Dreosti E. Wholebrain mapping of socially isolated zebrafish reveals that lonely fish are not loners. Elife. 2020;9. e55863. doi:10.7554/eLife.55863.

6. Galletly C. Psychiatry in the COVID-19 Era. UK: London, England: SAGE Publications Sage; 2020. doi: 10.1177/0004867420920359.

7. Rabiei A. 83\% of Iranians follow health protocols. Iran: Spokesman of the Government of the Islamic Republic of Iran; 2020, [updated 5/4/2020; cited 5/12/2020]. Available from: http://dolat.ir/detail/338621.

8. Herzberg MP, Hodel AS, Cowell RA, Hunt RH, Gunnar MR, Thomas KM. Risk taking, decision-making, and brain volume in youth adopted internationally from institutional care. Neuropsychologia. 2018;119:26270. doi: 10.1016/j.neuropsychologia.2018.08.022.

9. Isles AR, Winstanley CA, Humby T. Risk taking and impulsive behaviour: fundamental discoveries, theoretical perspectives and clinical implications. The Royal Society; 2019. doi: 10.1098/rstb.2018.0128.

10. Asadi-Pooya AA, Simani L. Central nervous system manifestations of COVID-19: A systematic review. J Neurol Sci. 2020:116832. doi: 10.1016/j.jns.2020.116832. 